\title{
SEARCHING FOR CIRCUMPLANETARY DISKS AROUND LkCa 15
}

\author{
Andrea Isella ${ }^{1}$, Clatre J. Chandler ${ }^{2}$, John M. Carpenter ${ }^{1}$, Laura M. Pérez ${ }^{2,3}$, And Luca Ricci ${ }^{1}$ \\ ${ }^{1}$ Department of Astronomy, California Institute of Technology, MC 249-17, Pasadena, CA 91125, USA; isella@ astro.caltech.edu \\ 2 National Radio Astronomy Observatory, P.O. Box 0, Socorro, NM 87801, USA \\ Received 2014 February 7; accepted 2014 April 22; published 2014 May 30
}

\begin{abstract}
We present Karl G. Jansky Very Large Array (VLA) observations of the $7 \mathrm{~mm}$ continuum emission from the disk surrounding the young star LkCa 15. The observations achieve an angular resolution of 70 mas and spatially resolve the circumstellar emission on a spatial scale of 9 AU. The continuum emission traces a dusty annulus of 45 AU in radius that is consistent with the dust morphology observed at shorter wavelengths. The VLA observations also reveal a compact source at the center of the disk, possibly due to thermal emission from hot dust or ionized gas located within a few AU from the central star. No emission is observed between the star and the dusty ring and, in particular, at the position of the candidate protoplanet LkCa $15 \mathrm{~b}$. By comparing the observations with theoretical models for circumplanetary disk emission, we find that if LkCa $15 \mathrm{~b}$ is a massive planet $\left(>5 M_{J}\right)$ accreting at a rate greater than $10^{-6} M_{J} \mathrm{yr}^{-1}$, then its circumplanetary disk is less massive than $0.1 M_{J}$, or smaller than 0.4 Hill radii. Similar constraints are derived for any possible circumplanetary disk orbiting within 45 AU from the central star. The mass estimates are uncertain by at least one order of magnitude due to the uncertainties on the mass opacity. Future ALMA observations of this system might be able to detect circumplanetary disks down to a mass of $5 \times 10^{-4} M_{J}$ and as small as $0.2 \mathrm{AU}$, providing crucial constraints on the presence of giant planets in the act of forming around this young star.
\end{abstract}

Key words: planet-disk interactions - protoplanetary disks - submillimeter: planetary systems - techniques: high angular resolution - techniques: interferometric

Online-only material: color figures

\section{INTRODUCTION}

High angular resolution observations at infrared and millimeter wavelengths have mapped in great detail nearby young ( $<5 \mathrm{Myr}$ ) circumstellar disks and revealed "holes" (Andrews et al. 2009, 2011; Brown et al. 2008, 2012; Cieza et al. 2012; Hughes et al. 2009; Isella et al. 2010a, 2010b, 2012; Mayama et al. 2012; Thalmann et al. 2010), asymmetric rings (Casassus et al. 2013; Fukagawa et al. 2013; Isella et al. 2013; Pérez et al. 2014; van der Marel et al. 2013), and spiral structures (Fukagawa et al. 2006; Garufi et al. 2013; Grady et al. 2013; Hashimoto et al. 2011; Muto et al. 2012) in the dust spatial distribution. These features suggest that the observed disks are perturbed by low mass companions which remain, to date, elusive.

Detecting giant planets and brown dwarfs orbiting at small separation from young stars with disks is indeed challenging. The variability of the photospheric lines in young stars and the presence of optically thick disks, prevent the use of radial velocities and transit techniques, respectively. Furthermore, direct imaging at optical and infrared wavelengths is feasible only if the companions have cleared the surrounding disk regions to expose themselves. However, even in this case, current high contrast cameras can image sub-stellar companions only at angular separations larger than about 0 '. 1 , which correspond to orbital radii larger than about $15 \mathrm{AU}$ at the distance of nearby star-forming regions (see, e.g., Garufi et al. 2013; Close et al. 2014). Alternatively, detections of sub-stellar companions orbiting within dustdepleted cavities have been obtained through near-infrared aperture masking interferometric observations (Biller et al. 2012; Huélamo et al. 2011; Kraus \& Ireland 2012), which achieve the telescope diffraction limit, e.g., 40 mas for a $10 \mathrm{~m}$ telescope at the wavelength of $2 \mu \mathrm{m}$. However, the sparse nature of aperture

\footnotetext{
3 Jansky Fellow.
}

masking measurements and the fact they provide information only on the closure phase of the Fourier transform of the surface brightness, introduce degeneracies in the reconstructions of the source emission.

In this work, we attempt to detect planets in the act of forming by observing the millimeter-wave thermal emission from their circumplanetary disks. Young giant planets embedded in their primordial nebula are expected to be surrounded by circumplanetary disks which regulate the angular momentum of the accreting material and provide the raw material to form moons. In analogy with circumstellar disks, circumplanetary disks are expected to intercept a large fraction $(>20 \%)$ of the optical and near-infrared radiation from the central planet and reemit it at longer wavelengths. Attempts to detect circumplanetary disks at millimeter wavelengths have been so far inconclusive. Greaves et al. (2008) reported the detection of a compact structure in the $1.3 \mathrm{~cm}$ continuum emission from HL Tau's disk at a radius of $65 \mathrm{AU}$, which is interpreted as the evidence of a circumplanetary disk with a mass of $14 M_{J}$. However, the rather low signal-to-noise of the detection and the lack of confirmation from observations at shorter wavelengths (Carrasco-González et al. 2009; Kwon et al. 2011) cast doubt on the real nature of the observed structure.

The target of our observations is LkCa 15, a 2-5 Myr old K5 $\operatorname{star}\left(L_{\star}=0.74 L_{\odot}, M_{\star}=1.0 M_{\odot}\right.$; Simon et al. 2000; Kenyon \& Hartmann 1995) located in the Taurus star-forming region at a distance of about $140 \mathrm{pc}$ (see, e.g., Loinard et al. 2007). The LkCa 15 circumstellar disk has a dust-depleted inner region of about 45 AU in radius (Piétu et al. 2006; Andrews et al. 2011; Isella et al. 2012). Despite this large cavity in dust, the star is accreting material from the disk at a rate of about $10^{-9} M_{\odot} \mathrm{yr}^{-1}$ (Hartmann et al. 1998). Kraus \& Ireland (2012) have reported the discovery of a candidate protoplanet, $\mathrm{LkCa} 15 \mathrm{~b}$, through aperture masking observations. The planet candidate is located 

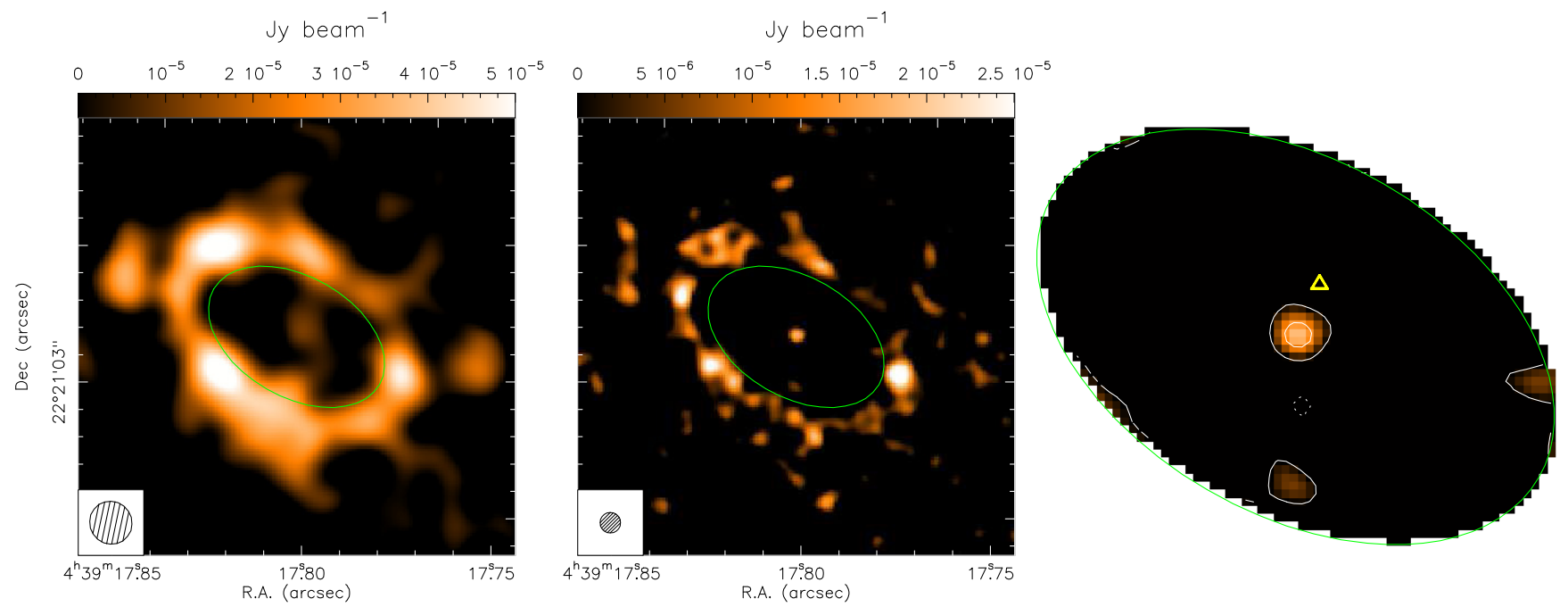

Figure 1. Left: 1". $6 \times 1$ 1". 6 map of LkCa 15's continuum disk emission observed at the wavelength of $7 \mathrm{~mm}$ obtained by reducing the weights of the complex visibilities measured on the longest baselines to increase the sensitivity of the extended structures. The rms noise level in the map is $6.1 \mu \mathrm{Jy} \mathrm{beam}^{-1}$. The FWHM of the synthesized beam is $0^{\prime \prime} 15$. Center: map of the $7 \mathrm{~mm}$ emission obtained by adopting natural weighting of the complex visibilities to maximize the angular resolution and the point source sensitivity. The rms noise level is $3.6 \mu \mathrm{Jy} \mathrm{beam}^{-1}$, and the FWHM of the synthesized beam is $0^{\prime \prime} 07$. The green ellipse corresponds to an orbital radius of $45 \mathrm{AU}$ and traces the outer edge of the dust-depleted cavity as measured from the observations at $1.3 \mathrm{~mm}$. Right: map of the innermost $45 \mathrm{AU}$ disk region. Contours are plotted at two and four times the noise level. The triangle shows the expected position of LkCa $15 \mathrm{~b}$ (Kraus \& Ireland 2012) assuming that the star is located at the peak of the $7 \mathrm{~mm}$ emission.

(A color version of this figure is available in the online journal.)

at a projected separation of 70 mas, which corresponds to a physical distance of $16 \mathrm{AU}$ if the planet orbit is in the plane of the circumstellar disk. Infrared photometric observations suggest a planet mass between 6 and $10 M_{J}$ (Kraus \& Ireland 2012).

Section 2 describes our Very Large Array (VLA) observations which seek to detect millimeter-wave emission from material orbiting around $\mathrm{LkCa} 15 \mathrm{~b}$, in addition to any circumplanetary disk within the dust-depleted cavity. A simple radiative transfer model for the circumplanetary disk emission is presented in Section 3, and the comparison with the observations is considered in Section 4. A short discussion of our results and the possibility of detecting circumplanetary disks with ALMA is presented in Section 5.

\section{OBSERVATIONS}

LkCa 15 was observed with the VLA in 2012 November, December, and 2013 January in the A configuration, which provides baselines between 0.7 and $36 \mathrm{~km}$ (project VLA/12B-196). The total observing time was $68 \mathrm{hr}$ and the time on-source was $21 \mathrm{hr}$. The data were obtained using four $2 \mathrm{GHz}$ intermediate frequencies (IFs) covering 39-47 GHz (8 GHz total bandwidth) using the $Q$-band receivers (see Perley et al. 2011, for an overview of the new capabilities of the VLA). The observations used the new 3 bit samplers, and indeed were used to help commission the hardware through the EVLA Commissioning Staff Observing program. The data were calibrated using the CASA data reduction package (e.g., McMullin et al. 2007), and a version of the VLA Calibration Pipeline scripts (see https://science.nrao.edu/ facilities/vla/data-processing/pipeline) modified to average data across each $2 \mathrm{GHz}$ IF to enable the use of a nearby, weak source ICRF J042655.7+232739 (0.2 Jy) as the complex gain calibrator. $3 \mathrm{C} 147$ was observed as the primary flux density calibrator (Perley \& Butler 2013), and 3C84 was observed as the bandpass calibrator. The absolute uncertainty in the overall flux density scale is estimated to be $10 \%$.
Figure 1 presents the maps of the $7 \mathrm{~mm}$ continuum emission observed toward LkCa 15 obtained using the CLEAN task in CASA with two different weighting schemes of the complex visibilities. The map on the left was obtained by reducing the weights of the longest baselines (tapering) to increase the sensitivity on the extended structures, while the central and right maps were obtained by adopting natural weighting to maximize the angular resolution and point source sensitivity. In this latter case, the observations achieve an angular resolution of 70 mas, or $9 \mathrm{AU}$ at the distance of the star, and a rms noise of $3.6 \mu \mathrm{Jy}$ beam $^{-1}$. The total flux density measured within the shown region is $0.52 \pm 0.06 \mathrm{mJy}$ and its consistent with the value of $0.44 \pm 0.17 \mathrm{mJy}$ measured by Rodmann et al. (2006) using the more compact D configuration. This suggest that although our observations are insensitive to spatial scales larger than $2^{\prime \prime}$, they recover most of dust emission. The spectral index of the millimeter wave emission $\left(F_{v} \propto v^{\alpha}\right)$ measured between $0.87 \mathrm{~mm}$ and $7 \mathrm{~mm}$ is $3.2 \pm 0.2$.

The observations reveal that most of the emission arises from an elliptical annulus characterized by a major axis of 0.28 , a minor axis of $0^{\prime \prime} .5$, and a position angle of about $60^{\circ}$, as measured from north toward east. The size and orientation of this annulus match the geometry of the dust emission observed at $0.87 \mathrm{~mm}$ and $1.3 \mathrm{~mm}$ (Piétu et al. 2006; Andrews et al. 2011; Isella et al. 2012), and confirm that LkCa 15's disk is depleted of dust within a radius of about $45 \mathrm{AU}$, as indicated by the green ellipse. In the tapered map, the dust emission is characterized by a clumpy structure on spatial scales comparable with the resolution of the observations, i.e., $20 \mathrm{AU}$. More precisely, the intensity varies azimuthally along the ring from 18 to $50 \mu \mathrm{Jy} \mathrm{beam}^{-1}$. The significance of these structures will be discussed in a forthcoming paper that studies the morphology of the dusty ring as revealed by the multi-wavelength continuum data.

The $7 \mathrm{~mm}$ map reveals a point source with a flux density of $16.6 \mu \mathrm{Jy}$ beam $^{-1}$ located at the center of the dusty ring. Because its coordinates are consistent with the proper motion corrected 
coordinates of $\mathrm{LkCa} 15$, we will assume in the following that this compact source pinpoints the position of the central star. The observations at shorter wavelengths do not detect any unresolved emission at the center of the disk. More precisely, Andrews et al. (2012) find that the disk regions within the dust-depleted cavity might contribute up to about $5 \mathrm{mJy}$ to the observed $870 \mu \mathrm{m}$ emission, while the $1.3 \mathrm{~mm}$ complex visibilities measured on baselines longer than $500 \mathrm{k} \lambda$ with CARMA set a $3 \sigma$ upper limit of $1.5 \mathrm{mJy}^{-1}$ beam $^{-1}$ for compact emission at the center of the disk (Isella et al. 2012). These measurements imply that the compact emission observed at $7 \mathrm{~mm}$ has a spectral index $\alpha<2.7$, which is smaller than spectral index derived for the spatially integrated disk emission.

The compact emission is not consistent with the radiation from the stellar photosphere, which has an expected $7 \mathrm{~mm}$ flux of $0.06 \mu \mathrm{Jy}$. Alternatively, we suggest that it might arise either from a narrow ring of millimeter size grains orbiting within a few AUs from the central star, or from ionized gas in the vicinity of the star (see, e.g., Panagia \& Felli 1974). In the former case, the measured flux would suggest the presence of about three Earth masses of small dust grains, as calculated by assuming optically thin emission, a dust temperature of $200 \mathrm{~K}$, and a dust opacity at $7 \mathrm{~mm}$ of $0.2 \mathrm{~cm}^{2} \mathrm{~g}^{-1}$. Investigating the nature of the compact emission requires high angular resolution observations at different wavelengths in order to measure its spectral index.

With the exception of the central source, we do not detect any emission above three times the noise level inside the dustdepleted cavity, and, in particular, at the expected position of LkCa 15 b (Kraus \& Ireland 2012). The constraints that this nondetection sets on the structure of circumplanetary disks orbiting within the dust-depleted cavity are discussed in the following sections.

\section{CIRCUMPLANETARY DISK MODEL}

We adopt a circumplanetary disk model characterized by a power-law surface density $\sigma(r) \propto r^{-p}$ that extends from the planet radius out to some outer radius $r_{d}$, at which the material is captured in orbit around the central star. Numerical simulations suggest that the truncation of a circumplanetary disk by the stellar gravitational field might occur between $0.1 r_{H}$ and $0.7 r_{H}$ (see, e.g., Canup \& Ward 2002; D’Angelo et al. 2002; Szulagyi et al. 2013; Ward \& Canup 2010), where $r_{H}=R_{p} \sqrt[3]{M_{p} / 3 M_{\star}}$ is the planet Hill radius, $R_{p}$ is the planet orbital radius, $M_{p}$ is the planet mass, and $M_{\star}$ is the mass of the central star. As in the analytical models of Canup \& Ward (2002), we define a nominal disk model with $p=3 / 4$. The effects that this choice has on the results are discussed in Section 4. For an inner disk radius $r_{\text {in }} \ll r_{d}$, the disk surface density can be integrated to give the total disk mass:

$$
M_{d}=2 \times 10^{-2}\left(\frac{\sigma_{1 \mathrm{AU}}}{36 \mathrm{~g} \mathrm{~cm}^{-2}}\right)\left(\frac{r_{d}}{1 \mathrm{AU}}\right)^{5 / 4} M_{J},
$$

where $\sigma_{1 \mathrm{AU}}$ is the surface density at $1 \mathrm{AU}$, and the normalization constant corresponds to the minimum mass with solar composition required to form the four largest moons of Jupiter, i.e., the Galilean satellites (Pollack \& Consolmagno 1984).

The temperature of the circumplanetary disk at the distance $r$ from the planet is expressed as

$$
T_{d}^{4}(r)=T_{\mathrm{irr}, \star}^{4}(R)+T_{\mathrm{irr}, p}^{4}(r)+T_{\mathrm{acc}}^{4}(r),
$$

where $T_{\mathrm{irr}, \star}$ corresponds to the irradiation from the central star at the orbital radius $R, T_{\mathrm{irr}, p}$ corresponds to the irradiation from the planet itself, and $T_{\text {acc }}$ is the contribution from the viscous dissipation within the circumplanetary disk. The disk temperature is calculated by extending the "two-layer" radiative transfer model developed by Chiang \& Goldreich (1997) in the context of circumstellar disks to the case of circumplanetary disks. The model assumes that the disk is in hydrostatic equilibrium between the gas pressure and the planet gravity, and the disk temperature is calculated by iterating on the vertical structure. The implementation of the model is the same as in Dullemond et al. (2001, see also Isella et al. 2009, 2010a, 2012) where the stellar effective temperature, radius, and luminosity are replaced by the values proper for the planet, and the circumstellar disk properties are replaced by the values corresponding to the circumplanetary disk.

The heating contribution from the planet depends on the planet effective temperature and radius, i.e., $T_{\mathrm{irr}, p}^{4} \propto T_{p}^{4}\left(r_{p} / r\right)^{2}$. We adopt Spiegel \& Burrows (2012) planetary models, which predict that the temperature and radius of a young planet depend on the formation process. In brief, planets formed by gravitational instability are expected to be hotter and more luminous than planets formed through the core accretion process. For the sake of simplicity, these two models are identified as "Hot start" and "Cold start," respectively. For example, a 2 Myr old $10 M_{J}$ planet is predicted to have $T_{p} \sim 2500 \mathrm{~K}$ and a $r_{p} \sim 1.7 R_{J}$ in the "Hot start" scenario, and $T_{p} \sim 700 \mathrm{~K}$ and $r_{p}=1.2 R_{J}$ for the "Cold start" case.

We assume that the circumplanetary disk is directly illuminated by the central star and calculate the stellar heating using the "two-layer" disk model where, this time, the source of radiation is the central star LkCa 15 . Following the result of Isella et al. (2012), we assume $T_{\mathrm{irr}, \star}(R)=40 \mathrm{~K} \times(R / 16 \mathrm{AU})^{-1 / 2}$.

Finally, the energy released per unit area in an annulus at distance $r$ from the planet by accreting material can be expressed as (D’Alessio et al. 1998)

$$
T_{\mathrm{acc}}^{4}(r)=\frac{3 G M_{p} M_{\mathrm{acc}}}{8 \pi \sigma_{\mathrm{SB}} r^{3}}\left[1-\left(\frac{r_{p}}{r}\right)^{1 / 2}\right],
$$

where $M_{\text {acc }}$ the mass accretion rate onto the planet and $\sigma_{\mathrm{SB}}$ is the Stefan-Boltzmann constant.

We show in Figure 2 the circumplanetary disk temperature calculated in the case of a $10 M_{J}$ planet characterized by effective temperatures of $2500 \mathrm{~K}$ and $700 \mathrm{~K}$, which are representative of the "Hot start" and "Cold start" models. The disk temperature is calculated for mass accretion rates of $0,10^{-6}, 10^{-4}$, and $10^{-2} M_{J} \mathrm{yr}^{-1}$. These latter three values correspond to the mean accretion rate required to build up a $10 M_{J}$ planet in $10^{7}, 10^{5}$, and $10^{3} \mathrm{yr}$ and might be representative of different accretion phases during the evolution of the planet and its parent disk. We find that for $M_{\mathrm{acc}} \geqslant 10^{-6} M_{J} \mathrm{yr}^{-1}$ the disk temperature is dominated by the accretion contribution expressed by Equation (3), and it is in first approximation independent from the assumed planet model. At $M_{\text {acc }} \geqslant 10^{-6} M_{J} \mathrm{yr}^{-1}$ the disk temperature can exceed the sublimation temperature of silicates grain which is around $1500 \mathrm{~K}$. In this case, and since the disk opacity at millimeter wavelengths is dominated by the dust, we adopt a disk inner radius $r_{\text {in }}$ consistent with the dust sublimation radius.

The disk emission is then calculated as

$$
\begin{aligned}
F_{7 \mathrm{~mm}}= & 2 \pi \cos i \int_{r_{\mathrm{in}}}^{r_{d}}\left\{1-\exp \left[\frac{-\sigma(r) \kappa_{7 \mathrm{~mm}}}{\cos i}\right]\right\} \\
& \times B_{\nu}\left[T_{d}(r)\right] \frac{r}{d^{2}} d r,
\end{aligned}
$$




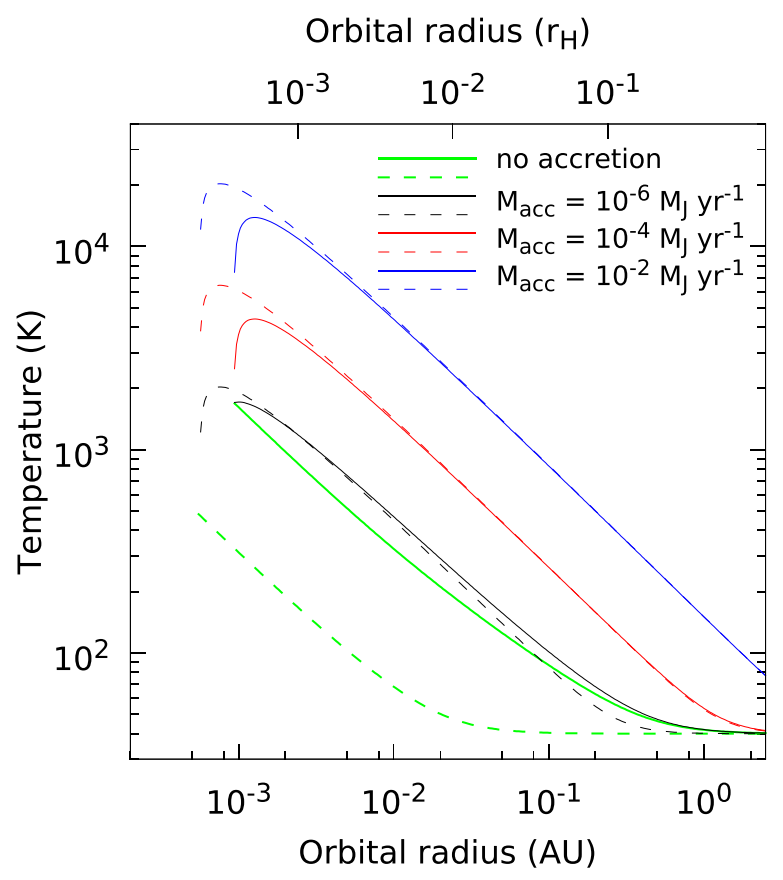

Figure 2. Interior temperature of a circumplanetary disk as a function of the distance from a $10 M_{J}$ planet orbiting at $16 \mathrm{AU}$ from $\mathrm{LkCa} 15\left(M_{\star}=1 M_{\odot}\right.$, $\left.L_{\star}=0.74 L_{\odot}\right)$. The solid and dashed curves correspond to planet effective temperatures of $2500 \mathrm{~K}$ and $700 \mathrm{~K}$, respectively. The different colors indicate models characterized by different mass accretion rates. From top to bottom: blue, $M_{\text {acc }}=10^{-2} M_{J} \mathrm{yr}^{-1}$; red, $M_{\mathrm{acc}}=10^{-4} M_{J} \mathrm{yr}^{-1}$; black, $M_{\mathrm{acc}}=$ $10^{-6} M_{J} \mathrm{yr}^{-1}$; green, no accretion. The viscous heating dominates over the irradiation from the planet and from the star for $M_{\mathrm{acc}} \geqslant 10^{-6} M_{J} \mathrm{yr}^{-1}$.

(A color version of this figure is available in the online journal.)

where the mass opacity $k_{7 \mathrm{~mm}}=0.002 \mathrm{~cm}^{2} \mathrm{~g}^{-1}$ is calculated by assuming a dust-to-gas ratio of 0.01 and a dust composition and grain size distribution as in Isella et al. (2012). We discuss the effects of relaxing this assumption in Section 5.

\section{RESULTS}

We adopt the model discussed above to calculate the emission of a possible disk orbiting around the candidate planet $\mathrm{LkCa}$ $15 \mathrm{~b}$, and then we generalize the results to the case of any circumplanetary disk within the $\mathrm{LkCa} 15$ 's disk dust-depleted cavity.

In the case of LkCa $15 \mathrm{~b}$, we assume a planet mass $M_{p}=$ $10 M_{J}$, and an orbital radius $R_{p}=16 \mathrm{AU}$ (Kraus \& Ireland 2012). We adopt a planet temperature $T_{p}=2500 \mathrm{~K}$ and a planet radius $r_{p}=1.7 R_{J}$, that correspond to the "Hot start" model for a 2 Myr old planet of $10 M_{J}$ (Spiegel \& Burrows 2012). The circumplanetary disk model has three free parameters: the disk outer radius, the disk mass, and the mass accretion rate. We assume a circumplanetary disk inclination of $45^{\circ}$, i.e., the same inclination of the circumstellar disk. We calculate the disk emission for disk outer radii between $0.2 \mathrm{AU}$ and $1.7 \mathrm{AU}$, corresponding to $0.1 r_{H}$ and $0.7 r_{H}$, respectively. Disk masses vary between $10^{-3} M_{p}$ and $M_{p}$, and the mass accretion rate ranges between $10^{-6}$ and $10^{-2} M_{J} \mathrm{yr}^{-1}$. In the next section we argue that $M_{\text {acc }}=10^{-6} M_{J} \mathrm{yr}^{-1}$ might be the most appropriate value for the $\mathrm{LkCa} 15$ system.

Because the circumplanetary disk radius is much smaller that the angular resolution of the our observations, we compare the theoretical $7 \mathrm{~mm}$ integrated disk flux to the point source sensitivity of the observations, i.e., $3.6 \mu \mathrm{Jy}$ beam $^{-1}$. The gray regions in Figure 3 show the disk models with $7 \mathrm{~mm}$ fluxes larger than two and three times the noise level of our observations. Given the non-detection at the position of $\mathrm{LkCa}$ $15 \mathrm{~b}$, these models are ruled out with a probability higher than $95.4 \%$ and $99.7 \%$, respectively. In general, the non-detection of LkCa $15 \mathrm{~b}$ disk rules out the presence of a large and massive circumplanetary disk. If the $7 \mathrm{~mm}$ disk emission is optically thin, the observations set an upper limit of the disk mass which is in first approximation independent of the disk radius and scales with the mass accretion rate as $M_{\mathrm{acc}}^{-1 / 4}$. The optically thin limit is shown by the almost horizontal lower margins of the gray regions in Figure 3. By contrast, if the $7 \mathrm{~mm}$ disk emission is optically thick, the observations set an upper limit on the disk outer radius that is independent on the disk mass and scales as $M_{\mathrm{acc}}^{-1 / 8}$. A variation of four orders of magnitude in $M_{\mathrm{acc}}$ therefore corresponds to a variation of a factor 3 in the upper limit for the outer disk radius for optically thick disks and of a factor 10 in upper limit on the disk mass in the optically thin case.

Figure 4 shows the constraints on the disk mass and radius for $M_{\mathrm{acc}}=10^{-6} M_{J} \mathrm{yr}^{-1}$ in the case of a colder planet (top panel)
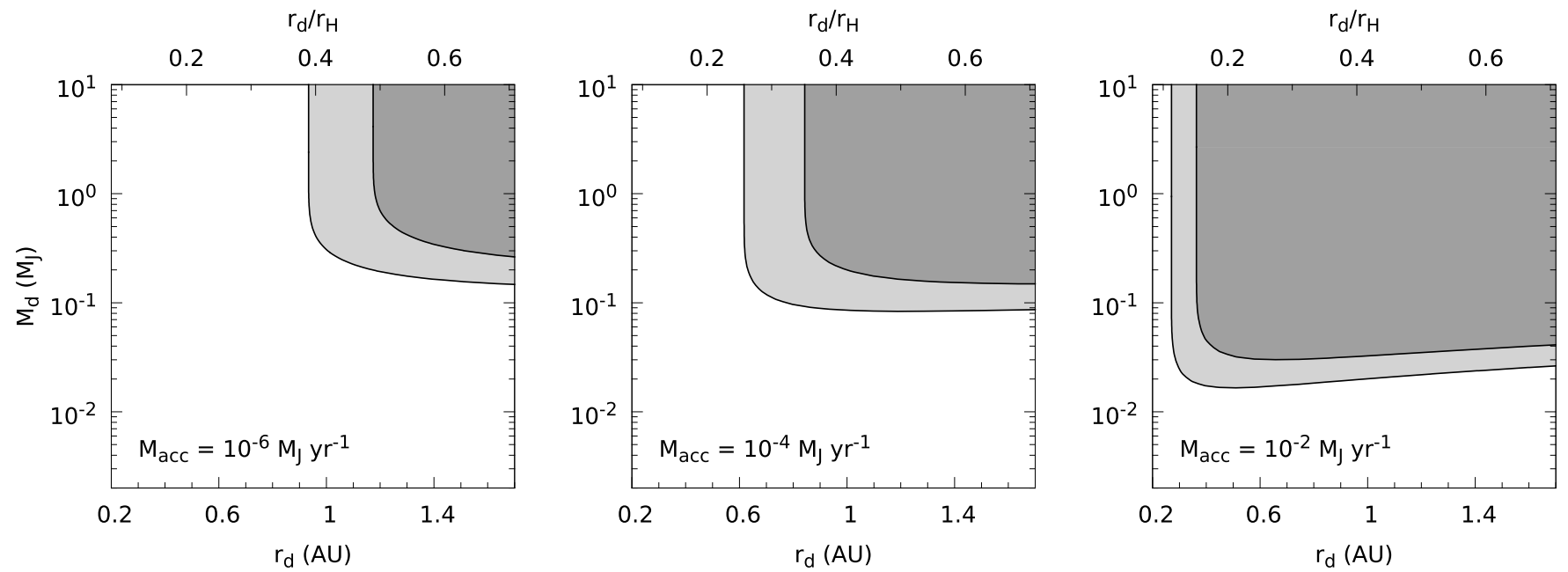

Figure 3. Light and dark gray regions show the disk models characterized by an integrated continuum flux at $7 \mathrm{~mm}$ larger than two and three times the noise level of our observations, i.e., $3.6 \mu \mathrm{Jy} \mathrm{beam}^{-1}$. Since the observations do not reveal any emission at the position of LkCa $15 \mathrm{~b}$, these circumplanetary disk models are ruled out at more than $95.4 \%$ and $99.7 \%$ confidence level, respectively. The disk emission has been computed for a planet characterized by $M_{p}=10 M_{J}, T_{p}=2500 \mathrm{~K}$, and $r_{p}=1.7 R_{J}$ and for mass accretion rates of $10^{-6}$ (left), $10^{-4}$ (middle), and $10^{-2} \mathrm{MJ}_{J} \mathrm{yr}^{-1}$ (right). 


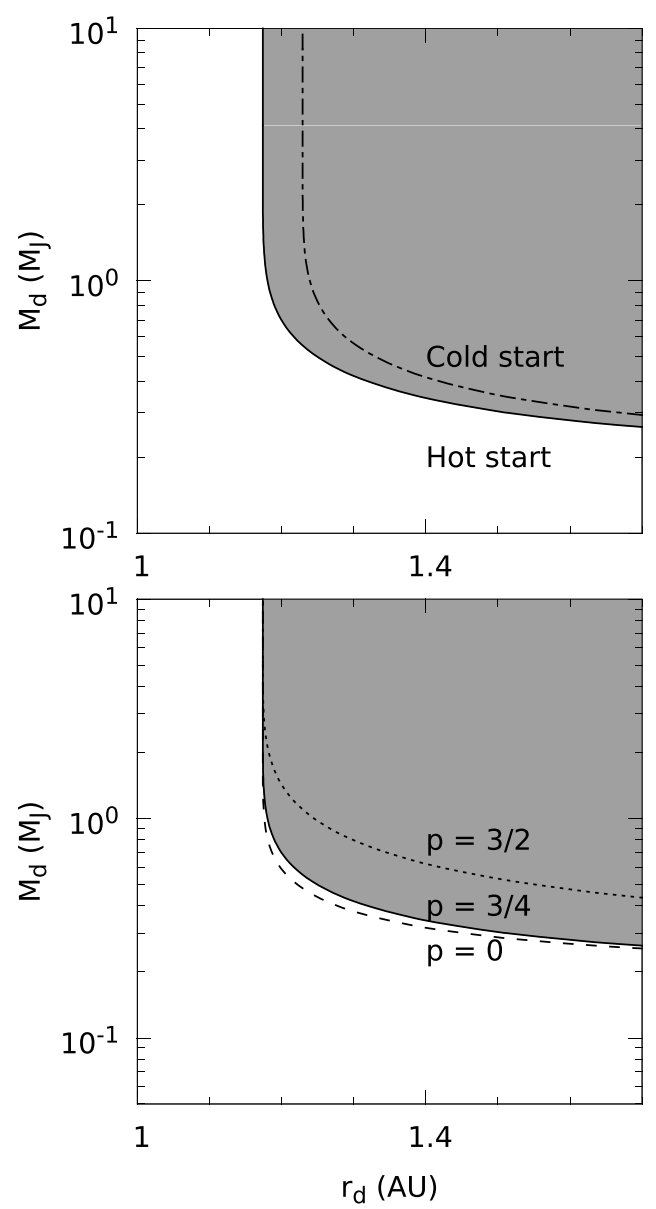

Figure 4. Dependence of the constraints on the LkCa 15 b circumplanetary disk structure on the planet model (top) and on the slope of the surface density (bottom). As in Figure 3, the dark gray region shows the model with integrated fluxes at $7 \mathrm{~mm}$ larger than three times the noise level of our observations. The top panel shows the comparison between the nominal "Hot start" model for LkCa $15 \mathrm{~b}\left(\right.$ age $=2 \mathrm{Myr}, M_{p}=10 M_{J}, T_{p}=2500 \mathrm{~K}$, and $\left.r_{p}=1.7 R_{J}\right)$ and a "Cold start" model (age $=2 \mathrm{Myr}, M_{p}=10 M_{J}, T_{p}=700 \mathrm{~K}$, and $r_{p}=1.2 R_{J}$ ) for $M_{\mathrm{acc}}=10^{-6} M_{J} \mathrm{yr}^{-1}$. The bottom panel compares the nominal disk model $(p=3 / 4)$ to the case of a flatter $(p=0)$ and steeper $(p=3 / 2)$ surface density profile.

and for different slopes of the disk surface density (bottom panel). Colder planets lead to a fainter millimeter disk emission, but as far as the disk temperature is dominated by the accretion heating, the planet temperature has a minor effect on the overall disk emission. We find that the disk flux is anti-correlated with the slope of the surface density, and, consequently, the upper limit on the disk mass diminishes for lower values of $p$. This is due to the fact that in the range of disk masses and radii shown in the figure, a large fraction of the $7 \mathrm{~mm}$ comes from the innermost optically thick regions of the disk. In this case, a steeper surface density implies that there is less mass in the outer optically thin region, which causes a decrement in the flux.

The results obtained for the LkCa $15 \mathrm{~b}$ disk can be generalized to giant planets with different masses and orbital radii by considering that (1) for $M_{\mathrm{acc}}>10^{-6} M_{J} \mathrm{yr}^{-1}$ the disk emission does not depend on the planet luminosity and (2) the heating contribution from the central star scales as $R^{-2}$. Circumplanetary disks orbiting closer to the star are therefore expected to be hotter and more luminous at millimeter wavelength, while disks orbiting further out should be colder and fainter. The overall effect on the constraints on the disk properties is shown in Figure 5, which compares the nominal model for $\mathrm{LkCa} 15 \mathrm{~b}$, to that of similar disks orbiting at 8 and 32 AU, i.e., half and twice the $\mathrm{LkCa} 15 \mathrm{~b}$ orbital radius, respectively. The effect of the orbital radius on the disk emission are larger for low mass accretion rate values and become negligible at $M_{\mathrm{acc}}=10^{-2} M_{J} \mathrm{yr}^{-1}$ since the disk temperature is totally dominated by the accretion heating.

\section{DISCUSSION AND CONCLUSIONS}

LkCa 15 is surrounded by a gas rich circumstellar disk that accretes onto the central star at a rate of $1.3 \times 10^{-9} M_{\odot} \mathrm{yr}^{-1}$, as measured by calculating the accretion luminosity from the ultraviolet excess emission over the stellar photosphere (Hartmann et al. 1998). Hydrodynamic simulations of giant planets embedded in gaseous rich circumstellar disks suggest that the amount of material accreting from the circumstellar disk onto a circumplanetary disk might be comparable to the mass accretion rate onto the central star. If circumplanetary disks have a viscosity similar to that of the circumstellar disks, then the mass accretion rate onto the planet should also be comparable to the mass accretion rate onto the central star (Zhu et al. 2011; Szulagyi et al. 2013). This suggests that giant planets orbiting inside the dust-depleted cavity might be accreting, in average, at a rate of $10^{-9} M_{\odot} \mathrm{yr}^{-1}$, or $10^{-6} M_{J} \mathrm{yr}^{-1}$. This value corresponds to the minimum mean mass accretion rate required to form a giant planet such as $\mathrm{LkCa} 15 \mathrm{~b}$ faster than the average disk dispersal time scale derived from infrared observations (Hernández et al. 2008). Higher accretion rates are predicted during the initial phase of planet formation (Ward \& Canup 2010; Shabram \& Boley 2013), while lower values are possible if circumplanetary disks are less viscous than the circumstellar disk. However, this would imply that any giant planet orbiting LkCa 15 has, in practice, accreted the majority of its final mass despite the young age of the system.

The non-detection of compact millimeter emission at the position of the candidate young planet $\mathrm{LkCa} 15 \mathrm{~b}$, and, in general, within the dust-depleted cavity in LkCa 15 circumstellar disk, sets upper limits on the mass and radius of possible circumplanetary disks. For $M_{\mathrm{acc}} \geqslant 10^{-6} M_{J} \mathrm{yr}^{-1}$, we find that the disk temperature is dominated by the viscous heating released by the accreting material and the $7 \mathrm{~mm}$ continuum disk emission is in first approximation independent of the luminosity of the central planet. Under this condition, our observations exclude the presence of disks more massive than about $0.1 M_{J}$ and larger than about $1 \mathrm{AU}$, or $0.4 r_{H}$ for a $10 M_{J}$ planet orbiting at $16 \mathrm{AU}$ from the central star. Higher mass accretion rates would imply lower disk masses and radii, but, as discussed in the previous section, an increase of four orders of magnitude in the mass accretion rate would lower the constraints on disk mass only by a factor of 10 and the disk radius by a factor of 3 . If the dust-depleted cavity observed in the LkCa 15 disk originates from the dynamical clearing operated by brown dwarfs or massive planets (e.g., $>10 M_{J}$ ), our VLA observations suggest that the mass of their circumstellar disks should be less than a few percent of the planetary mass, unless they have very small radii.

The upper limits for the circumplanetary disk mass are derived adopting the dust model employed in the study of the LkCa 15 circumstellar disk emission, which assumes a grain composition as in Pollack et al. (1994), a grain size distribution $n(a) \propto a^{-3.5}$ ranging between $0.5 \mu \mathrm{m}$ and $0.5 \mathrm{~mm}$, and a gas-to-dust ratio of 100 (Isella et al. 2012). The corresponding mass opacity at $7 \mathrm{~mm}$ is $2 \times 10^{-3} \mathrm{~cm}^{2} \mathrm{~g}^{-1}$. There are however at least two (competing) physical processes that might cause the mass opacity of a circumplanetary disk to differ from that of the 

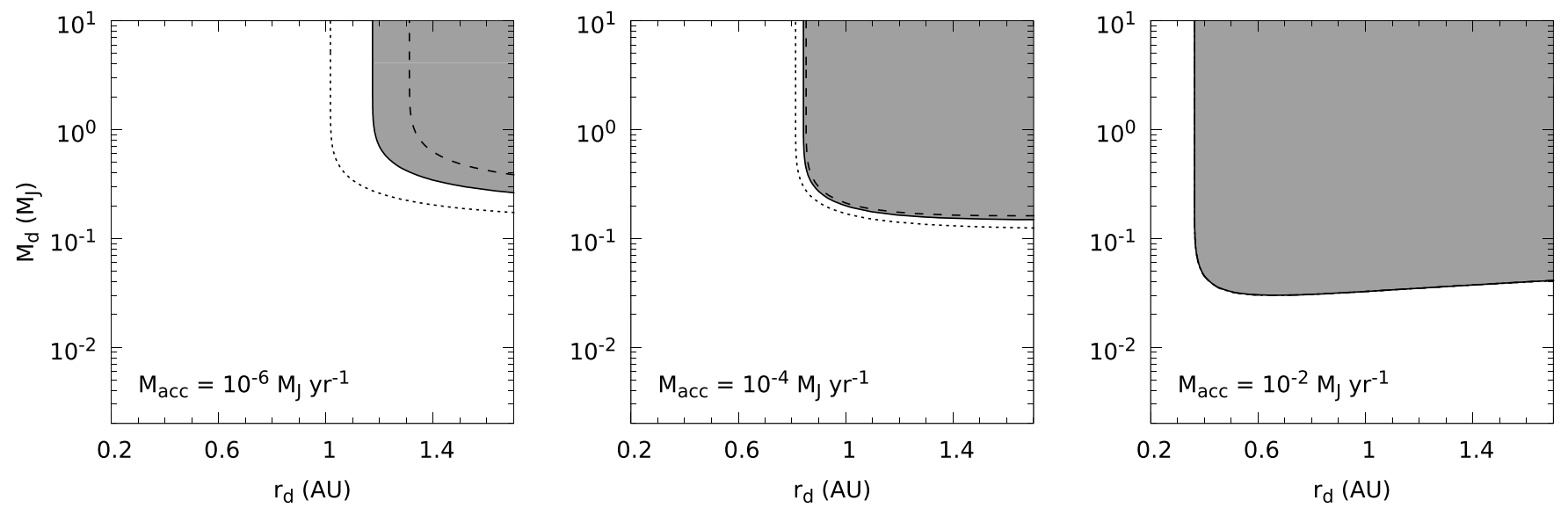

Figure 5. Dependence of the constraints on the circumplanetary disk structure on the planet orbital radius. As in Figure 3, the dark gray region shows the model with integrated fluxes at $7 \mathrm{~mm}$ larger than three times the noise level of our observations in the case of circumstellar disk orbiting at $16 \mathrm{AU}$ from the central star. The dotted and dashed curves indicate circumstellar disks with $7 \mathrm{~mm}$ fluxes equal to three times the noise level orbiting at $8 \mathrm{AU}$ and $32 \mathrm{AU}$ from the central star, respectively.

parent circumstellar disk. The first process is the filtration, or trapping, of large dust grains at the outer edge of the cavity cleared by the gravitational interaction with massive planets. More precisely, Zhu et al. (2011) find that only dust grain smaller than $10-100 \mu \mathrm{m}$ might be able to filtrate from the outer disk into the dust-depleted cavity. In this scenario, the material accreting onto circumplanetary disks would be depleted by large dust grains and have a gas-to-dust ratio higher than the circumstellar disk material. For example, if all the grains larger than $10 \mu \mathrm{m}$ are trapped in the outer disk at $R>45 \mathrm{AU}$, then the material accreting onto the circumplanetary disk would have a gas-todust ratio of 1000 , i.e., 10 times larger than the material in the outer disk. The resulting mass opacity at $7 \mathrm{~mm}$ calculated accounting for both the higher gas-to-dust ratio and for the reduced maximum grain size would be $1.6 \times 10^{-4} \mathrm{~cm}^{2} \mathrm{~g}^{-1}$. However, the dust grains in a circumplanetary disk grains are expected to interact and grow in size similarly to what happens in the innermost regions of circumstellar disks. Actually, grain growth in circumplanetary disks is a key ingredient in current models for the formation of the Galilean satellites (see, e.g., Canup \& Ward 2009). In this case, if the maximum grain size grows, for example, from $10 \mu \mathrm{m}$ to $1 \mathrm{~cm}$, then the $7 \mathrm{~mm}$ mass opacity would increase to $1.8 \times 10^{-3} \mathrm{~cm}^{2} \mathrm{~g}^{-1}$ (now assuming a gas-to-dust ratio of 1000). Given the uncertainties on mass opacity, the constraints in the circumplanetary disk mass set by our observations should therefore be considered uncertain by at least one order of magnitude.

Future ALMA observations will provide better constraints on the mass and radius of circumplanetary disks orbiting around $\mathrm{LkCa} 15$, and, in general, within disks characterized by large dust-depleted cavities. ALMA full array will achieve in a few hours a sensitivity in disk mass 100 times greater than that obtained by VLA observations (Figure 6) and an angular resolution sufficient to detect circumplanetary disks orbiting at a few $\mathrm{AU}$ from the central star at the distance of $140 \mathrm{pc}$. Assuming a dust emissivity proportional to $v^{1.0}$, ALMA band 7 observations $(\lambda=850 \mu \mathrm{m})$ will achieve a mass sensitivity of $5 \times 10^{-4} M_{J}$ in $1 \mathrm{hr}$ of integration on source. Furthermore, ALMA observations in the other bands, although less sensitive, will allow the measurements of the spectral index of the continuum emission to study the evolution of solids, in a manner similar to that employed to study the grain size distribution in circumstellar disks (see, e.g., the review by Testi et al. 2014).

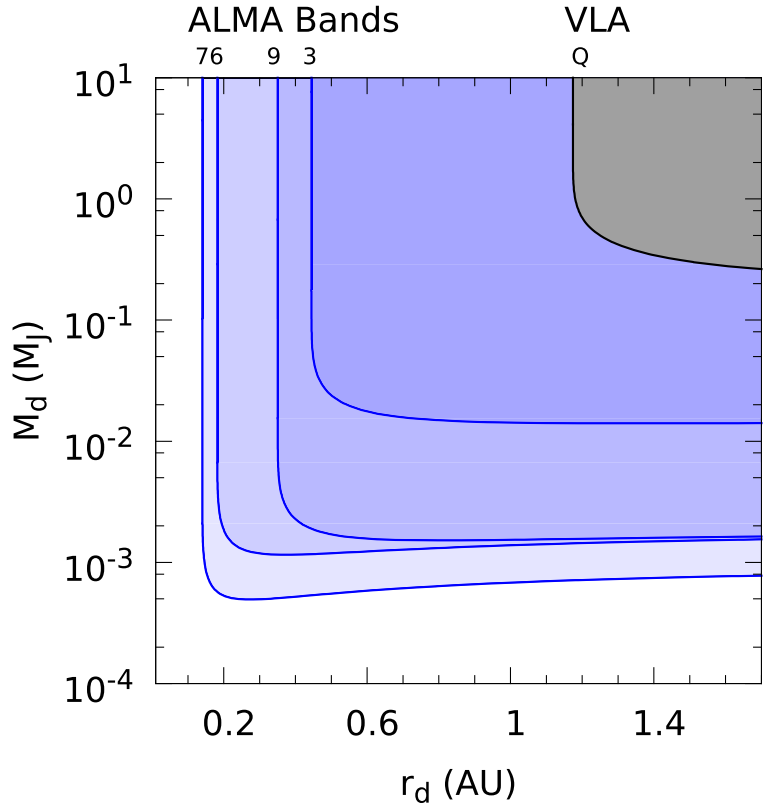

Figure 6. Parameter space for the mass and radius of $\mathrm{LkCa} 15$ b's circumplanetary disk that can be probed at more that $99.7 \%$ confidence level in $1 \mathrm{hr}$ of ALMA observations in bands $3(110 \mathrm{GHz}), 6(230 \mathrm{GHz}), 7(345 \mathrm{GHz})$, and 9 $(650 \mathrm{GHz})$. The disk emission was calculated by assuming a mass accretion rate of $10^{-6} M_{J} \mathrm{yr}^{-1}$ and a dust opacity emissivity proportional to $v^{1.0}$. The gray region corresponds to the region probed by our VLA observations, and it is the same as in the left panel of Figure 3.

(A color version of this figure is available in the online journal.)

A.I., L.P., and J.M.C. acknowledge support from NSF award AST-1109334. The National Radio Astronomy Observatory is a facility of the National Science Foundation operated under cooperative agreement by Associated Universities, Inc.

\section{REFERENCES}

Andrews, S. M., Rosenfeld, K. A., Wilner, D. J., \& Bremer, M. 2011, ApJL, 742, L5

Andrews, S. M., Wilner, D. J., Hughes, A. M., Qi, C., \& Dullemond, C. P. 2009, ApJ, 700, 1502

Andrews, S. M., Wilner, D. J., Hughes, A. M., et al. 2012, ApJ, 744, 162

Biller, B., Lacour, S., Juhász, A., et al. 2012, ApJL, 753, L38

Brown, J. M., Blake, G. A., Qi, C., Dullemond, C. P., \& Wilner, D. J. 2008, ApJL, 675, L109 
Brown, J. M., Rosenfeld, K. A., Andrews, S. M., Wilner, D. J., \& van Dishoeck, E. F. 2012, ApJL, 758, L30

Canup, R. M., \& Ward, W. R. 2002, AJ, 124, 3404

Canup, R. M., \& Ward, W. R. 2009, in Origin of Europa and the Galilean Satellites, ed. R. T. Pappalardo, W. B. McKinnon, \& K. K. Khurana (Tucson, AZ: Univ. Arizona Press), 59

Carrasco-González, C., Rodríguez, L. F., Anglada, G., \& Curiel, S. 2009, ApJL, 693, L86

Casassus, S., van der Plas, G., M, S. P., et al. 2013, Natur, 493, 191

Chiang, E. I., \& Goldreich, P. 1997, ApJ, 490, 368

Cieza, L. A., Mathews, G. S., Williams, J. P., et al. 2012, ApJ, 752, 75

Close, L. M., Follette, K. B., Males, J. R., et al. 2014, ApJL, 781, L30

D’Alessio, P., Canto, J., Calvet, N., \& Lizano, S. 1998, ApJ, 500, 411

D’Angelo, G., Henning, T., \& Kley, W. 2002, A\&A, 385, 647

Dullemond, C. P., Dominik, C., \& Natta, A. 2001, ApJ, 560, 957

Fukagawa, M., Tamura, M., Itoh, Y., et al. 2006, ApJL, 636, L153

Fukagawa, M., Tsukagoshi, T., Momose, M., et al. 2013, PASJ, 65, L14

Garufi, A., Quanz, S. P., Avenhaus, H., et al. 2013, A\&A, 560, A105

Grady, C. A., Muto, T., Hashimoto, J., et al. 2013, ApJ, 762, 48

Greaves, J. S., Richards, A. M. S., Rice, W. K. M., \& Muxlow, T. W. B. 2008, MNRAS, 391, L74

Hartmann, L., Calvet, N., Gullbring, E., \& D’Alessio, P. 1998, ApJ, 495, 385

Hashimoto, J., Tamura, M., Muto, T., et al. 2011, ApJL, 729, L17

Hernández, J., Hartmann, L., Calvet, N., et al. 2008, ApJ, 686, 1195

Huélamo, N., Lacour, S., Tuthill, P., et al. 2011, A\&A, 528, L7

Hughes, A. M., Andrews, S. M., Espaillat, C., et al. 2009, ApJ, 698, 131

Isella, A., Carpenter, J. M., \& Sargent, A. I. 2009, ApJ, 701, 260

Isella, A., Carpenter, J. M., \& Sargent, A. I. 2010a, ApJ, 714, 1746

Isella, A., Natta, A., Wilner, D., Carpenter, J. M., \& Testi, L. 2010b, ApJ, 725, 1735

Isella, A., Pérez, L. M., \& Carpenter, J. M. 2012, ApJ, 747, 136

Isella, A., Pérez, L. M., Carpenter, J. M., et al. 2013, ApJ, 775, 30

Kenyon, S. J., \& Hartmann, L. 1995, ApJS, 101, 117

Kraus, A. L., \& Ireland, M. J. 2012, ApJ, 745, 5
Kwon, W., Looney, L. W., \& Mundy, L. G. 2011, ApJ, 741, 3

Loinard, L., Torres, R. M., Mioduszewski, A. J., et al. 2007, ApJ, 671, 546

Mayama, S., Hashimoto, J., Muto, T., et al. 2012, ApJL, 760, L26

McMullin, J. P., Waters, B., Schiebel, D., Young, W., \& Golap, K. 2007, in ASP Conf. Ser. 376, Astronomical Data Analysis Software and Systems XVI, ed. R. A. Shaw, F. Hill, \& D. J. Bell (San Francisco, CA: ASP), 127

Muto, T., Grady, C. A., Hashimoto, J., et al. 2012, ApJL, 748, L22

Panagia, N., \& Felli, M. 1974, MmSAI, 45, 377

Pérez, L. M., Isella, A., Carpenter, J. M., \& Chandler, C. J. 2014, ApJ, 783, L13

Perley, R. A., \& Butler, B. J. 2013, ApJS, 206, 16

Perley, R. A., Chandler, C. J., Butler, B. J., \& Wrobel, J. M. 2011, ApJL, 739, L1

Piétu, V., Dutrey, A., Guilloteau, S., Chapillon, E., \& Pety, J. 2006, A\&A, 460, L43

Pollack, J. B., \& Consolmagno, G. 1984, in Origin and Evolution of the Saturn System, ed. T. Gehrels \& M. S. Matthews (Tucson, AZ: Univ. Arizona Press), 811

Pollack, J. B., Hollenbach, D., Beckwith, S., et al. 1994, ApJ, 421, 615

Rodmann, J., Henning, T., Chandler, C. J., Mundy, L. G., \& Wilner, D. J. 2006, A\&A, 446, 211

Shabram, M., \& Boley, A. C. 2013, ApJ, 767, 63

Simon, M., Dutrey, A., \& Guilloteau, S. 2000, ApJ, 545, 1034

Spiegel, D. S., \& Burrows, A. 2012, ApJ, 745, 174

Szulagyi, J., Morbidelli, A., Crida, A., \& Masset, F. 2014, ApJ, 782, 65S

Testi, L., Birnstiel, T., Ricci, L., et al. 2014, in Protostars and Planets VI, ed. H. Beuther, R. Klessen, C. Dullemond, \& Th. Henning (Tucson, AZ: Univ. Arizona Press), in press (arXiv:1402.1354)

Thalmann, C., Grady, C. A., Goto, M., et al. 2010, ApJL, 718, L87

van der Marel, N., van Dishoeck, E. F., Bruderer, S., et al. 2013, Sci, 340, 1199

Ward, W. R., \& Canup, R. M. 2010, AJ, 140, 1168

Zhu, Z., Nelson, R. P., Hartmann, L., Espaillat, C., \& Calvet, N. 2011, ApJ, 729,47 